\title{
OBESITY IN PRESCHOOL CHILDREN
}

\author{
Oana Teslariu', Carmen Oltean ${ }^{2}$, Laura Alexandra Gavriluta ${ }^{3}$, \\ Maria Liliana Iliescu ${ }^{4}$, Dana-Teodora Anton-Paduraru ${ }^{1}$ \\ ${ }^{1} 3^{\text {rd }}$ Clinic of Pediatrics, "Gr. T. Popa" University of Medicine and Pharmacy, Iasi \\ 2"Sf. Maria" Children's Emergency Hospital, Iasi \\ 3 "Gr. T. Popa" University of Medicine and Pharmacy, Iasi \\ ${ }^{4}$ Public Health and Sanitary Management, \\ "Gr. T. Popa" University of Medicine and Pharmacy, Iasi
}

\begin{abstract}
Primary obesity in children, one of the most common nutrition diseases in the world, shows particularities depending on the age regarding the risk factors, prevention and treatment. The aim of the study was the assessment of preschool patients diagnosed with primary obesity in the 3rd Clinic of Pediatrics, "Sf. Maria" Children's Emergency Hospital lasi, in order to identify the onset of overweight/obesity and the prenatal and postnatal risk factors from the first 6 years of life involved in causing obesity. We conclude that in order to combat obesity the emphasis should be on prevention, particularly important being the measures that promote healthy eating and combat sedentariness.
\end{abstract}

Keywords: obesity, preschool children, alimentary excess, sedentariness

\section{INTRODUCTION}

In the last decades, obesity - a complex and multifactorial disease, has become one of the most common nutritional diseases in the world, having a pandemic extent, so that World Health Organization (WHO) considers it "the disease of 21 th century". According to a study conducted in 79 countries, WHO estimated that there are 250 million obese people worldwide, of which 22 million are children under the age of $5(1,2)$.

Obesity among preschool children is a serious public health problem worldwide, especially due to the consequences it may have on long-term health. Recent studies have demonstrated that, in most cases, early childhood obesity onset could evolve into adulthood, thus increasing the risk of diseases affecting the quality and length of life, such as cardiovascular diseases, type 2 diabetes, orthopedic complications, psycho-behavioral pathology (3). The risk of children with obesity early in life to be- come obese adults is of $80 \%$ for those whose both parents are obese, and $40 \%$ for those with only one obese parent.

The physical and psychological consequences, the enormous costs and the difficulties encountered during the treatment of obesity, clearly indicate the need for prevention as a public health target $(4,5)$. The identification of the risk factors is a key element in reducing the incidence of obesity in childhood (6). Evidence shows that a combination of dietary intervention, behavioral therapy and exercise has a significant impact in reducing body weight of obese and overweight children $(7,8)$.

The objective of this study was the assessment of preschool patients diagnosed with primary obesity in the $3^{\text {rd }}$ Clinic of Pediatrics Clinic - "Sf. Maria" Children's Emergency Hospital Iasi, in order to identify the onset of overweight/obesity and the prenatal and postnatal risk factors from the first 6 years of life involved in causing obesity. 


\section{MATERIAL AND METHODS}

A retrospective study was conducted over a period of 3 years (2012-2014), on a group of 33 patients of preschool age (3-6 years old) diagnosed with primary obesity in the $3^{\text {rd }}$ Clinic of Pediatrics, "Sf. Maria" Children's Emergency Hospital Iasi. The study protocol included:

Criteria for inclusion in the study: body mass index (BMI) above the 95th percentile, exclusion of obesity belonging to a genetic syndrome, endocrynopathies or psychosomatic diseases.

General anamnesis: age, sex, environment (urban/rural), birth weight, nutrition and growth rate in the first year of life, age at the onset of excessive weight gain, precipitating circumstances, presence of other previous severe chronic diseases.

Family medical history: presence of obesity or other disorders induced or aggravated by obesity (hypertension, cardiovascular disease, diabetes, endocrine diseases), mother's opinion regarding alimentation, the meaning of a healthy child, quantities of foods that the child should receive, eating habits).

Obesity history: onset and apparent trigger circumstances, previous investigations and therapeutic approach, previous test results and the reason for the treatment failure.

Food survey: assessment of major food groups intake and frequency of food intake from each nutritional group based on mother's statements, excess of carbohydrates, calories intake variations from day to day, calories distribution for each meal, assessment of caloric and protein intake compared to the usual nutritional recommendations.

Physical activity survey: the number of hours of sleep, the distance from home to kindergarten, the number of hours of free activity (games, walking), the number of hours spent daily on TV and computer, physical activity practiced together with the family.

The general clinical examination of the patients was completed with the measurement of the anthropometric parameters (weight, height, body mass index).

\section{RESULTS}

The 33 patients with primary obesity represented $1.46 \%$ of the hospitalized children with the same age in the studied period. Analyzing the gender distribution, study results confirmed data from literature, the female being predominant (female: male = 1.38: 1). Most of the investigated patients (64\%) lived in urban areas, where consumption of hypercaloric drinks and food, but with low nutritional value, is higher.

Measurement results of anthropometric parameters led to the framing of the 33 cases in different degrees of obesity (Fig. 1):

- obesity grade I (mild ): 17 cases (52\%)

- obesity grade II (moderate): 9 cases (27\%)

- obesity grade III (severe): 7 cases (21\%)

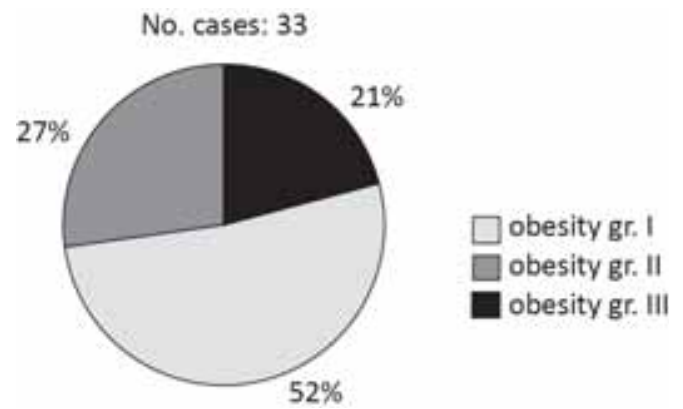

FIGURE 1. Group distribution depending on the degree of obesity

In most cases, the onset of excessive weight gain occurred at age of 3 years (49\%), followed by infancy $(30 \%)$. Periods of rapid weight gain were related to: convalescence of infectious diseases 3 cases (9\%), drug administration (prednisone) 2 cases $(6 \%)$, sedentariness -4 cases $(12 \%)$, hyperphagia and vicious habits -21 cases $(64 \%)$. In 3 cases $(9 \%)$ there were not identified no "noticeable elements" in the pathogenesis of the disease.

The medical history revealed that 2 of the patients had low birth weight (BW) and 6 high weight $(4,000 \mathrm{~g})$. Literature data confirm that a BW > 4,000 $\mathrm{g}$ is associated with an increased risk for obesity in both childhood and adult age, compared with patients who had a BW below 4,000 g. In the same time, BW under 2,500 $\mathrm{g}$ is associated with a decreased risk of developing overweight compared to people with $\mathrm{BW}>2,500 \mathrm{~g}(9-12)$.

Correlating weight with height, it was noticed that 21 cases (64\%) showed an accelerated growth both in weight and height, the weight growth exceeding proportionally the height growth. 12 cases $(36 \%)$ presented excess weight compared to a normal stature.

The alarming increase of child obesity imposed the identifying of the factors that contribute to early excess weight, particularly in high-risk groups, including preschool age (13).

10 cases $(30 \%)$ had a family history of obesity (Table 1). In 5 of the 10 families where obesity was present, the parents also presented other medical conditions induced or aggravated by obesity: isch- 
emic heart disease (1 case), hypertension ( 2 cases), diabetes type II ( 1 case), asthma (1 case).

TABLE 1. The distribution of cases by family history

\begin{tabular}{|c|c|c|c|}
\hline \multicolumn{2}{|c|}{ Family history } & No. cases & $\%$ \\
\hline \multicolumn{2}{|c|}{ Negative } & 23 & 70 \\
\hline \multirow[t]{2}{*}{ Positive } & one obese parent & 7 & 21 \\
\hline & both obese parents & 3 & 9 \\
\hline
\end{tabular}

Family survey concerning parents opinion about how much their children should eat, revealed excess food in $64 \%$ of cases. In some families (15 cases $-45 \%$ ) children were forced to eat the entire quantity of food ("empty plate syndrome"), this habit being a psychological pressure from parents.

Analysis of eating habits revealed that within 27 families $(82 \%)$ the richest caloric meal was dinner, when it was consumed concentratefoods, rich in carbohydrates and fat (fries, pasta, pork, concentrated sweets, mayonnaise).

Food survey showed that only 3 of 33 patients included in the studied group received natural diet for 4 months, the rest receiving mixt diet or artificial milk. It is known that breast milk, for at least the first 4 months of life, is a protective factor in the occurrence of obesity (16). An important number of cases $(60 \%)$ received improper dilutions of cow's milk or too high concentrations of milk powder. In 14 cases $(42 \%)$ the principles of diversification were not complied with.

The medical history revealed that only in 5 cases $(15 \%)$ the family addressed previously to the doctor for dietary and physical activity recommendations. The therapy failed in all the cases because the parents did not respect the recommendations.

Food survey carried out thoroughly allowed the assessment of food intake frequency of the main nutritional groups. Thus, an unbalanced diet was revealed with excessive consumption of dairy (milk, yogurt, cheese, butter) -7 cases $(21 \%)$, meat (pork, beef, poultry) - 15 cases (45\%), eggs -10 cases $(30 \%)$, fruits -5 cases $(15 \%)$, bread and other flour products (biscuits, macaroni, pasta, rice) -26 cases $(79 \%)$, juices -15 cases $(45 \%)$.

In all cases it was revealed a continuously excessive intake of dietary calories, mainly from carbohydrate consumption. Following the calorie intake changes from day to day, it was observed in most cases an increased calorie intake almost daily. Following the distribution of calorie intake during the day, the richest meal in calories was dinner.

To test the diet's caloric imbalance (between intake and energy consumption) and to modify the behavior towards calorie intake, the evaluation of the 33 patients contained an assessment of physical activity during 24 hours:

- number of hours of sleep (average): day $1 \mathrm{hr}$. 30 minutes, night -10 hours

- the way of travel from home to kindergarten: walking -7 cases; means of transport -18 cases

- number of hours of free activity (walking, playing)/day: 1 hour - 10 cases (30\%), 2 hours -7 cases (2\%), 3 hours -3 cases $(9 \%)$

- number of hours spent daily (average) in front of the TV or the computer: 3 hours.

Only 5 patients were participating in physical activities with family (running, rides - tricycle/bicycle or roller skates).

The treatment given in the 33 cases of obesity consisted of a dietary regime that covered 1,200 calories/day correlated with a program of physical activity, psychotherapy and education of parents (providing information regarding foods composition, foods that should be eaten in limited quantities but taking into account the child preferences, participation in outdoor attractive games with the parents or in small sport competitions that bring them satisfaction). At the individual level, the targeted treatment on dietary and physical activity was found to have the highest rate of efficacy, because the child's behavior is still malleable (20). At the same time, improvement was noticed in the condition of the patients, along with the increase of life quality.

\section{DISCUSSIONS}

Increased height growth delimits the obesity caused by alimentary excess from the symptomaticobesity of genetic syndromes and endocrynopathies, in which case the clinical examination associates specific signs and symptoms (dysmorphia, retardation).

Early onset of obesity is strongly correlated with the hereditary inheritance and epigenetic factors, under the influence of environmental factors "in utero" and postnatally.

The increasing global prevalence of obesity and overweight among children is due, on one hand, to the increased energy intake, especially of foods with high energy-density, high in fat and sugar, and on the other hand, to the decreased physical activity due to sedentariness growth.

Parent implication in terms of food excess is essential, overweight mothers having the tendency to provide children with larger portions and higher fat content (14). 
Children are likely to be inactive in the evening after dinner in front of the TV or computer, and thus the excess of calories is not consumed (15). In all cases from our study group an unbalanced diet was highlighted concerning the main nutrients, and in all cases children consumed hypercaloric snacks (sweets, soft drinks) between meals.

The increasing frequency of artificial nutrition after birth (excess of carbohydrates and high osmotic charge of formula milk), early introduction of solid foods (early diversification), increased use of commercial food in diversified diet (most of them unbalanced, with high carbohydrate intake and low protein intake), led to an excessive weight increase of infants and excessive storage of fat. It is known that parental factors, such as low education, offering sweets as a reward, lack of time, insufficient information regarding a healthy diet, plays an important role in the diet of young children (17).

Several studies have suggested a positive correlation between excessive food intake and obesity in children, even when controlling other factors such as exercise and body weight of parents (19).

After the assessment of the physical activity during 24 hours, the patients received recommendations for adjusting the physical activity, and advices for the introduction of additional physical activity leading to increased energy consumption.

Introducing exercise into daily routine and in the preschool curriculumhas been shown to have beneficial effects in reducing the incidence of obe- sity (21). An important role is played by the involvement of the family, because attitudes, perceptions and behavior of parents can influence the development of children's body weight (22).

\section{CONCLUSIONS}

High birth weight may be a risk factor for the development of obesity in preschool children.

The obesity of the preschoolers from the study group was exogenous, by hyperphagia, involving at the same time a sedentary lifestyle and vicious eating habits, with an important imbalance of dietary calories.

For preschoolers with obesity, family cooperation is very important in order to achieve an appropriate diet and to maintain an active lifestyle.

The emphasis in combating child obesity should be moved towards its prevention, particularly important being the measures to promote healthy eating andavoiding sedentariness.

Obesity prevention requires the implication of all the elements involved in parents and children education (doctors, school, media), regarding the eating habits and an active life style, starting at small ages.

The treatment of moderate and severe obesity is difficult and it requires a multidimensional approach (educators, nutritionists, pediatricians, psychologists, trainers, coaches) in order to reduce the body mass index by "small steps".

\section{REFERENCES}

1. Rodriguez G., Pietrobelli A., Wang Y. et al. Methodological aspects for childhood and adolescence obesity epidemiology. Epidemiology of obesity in children and adolescents - prevalence and etiology. New York, USA: Springer Publisher, 2011: 21-40.

2. Veugelers P.J., Fitzgerald A.L. Prevalence of and risk factors for childhood overweight and obesity. CMAJ 2005; 173(6): 607-613.

3. Manios Y., Costarelli V., Kolotourou M. et al. Prevalence of obesity in preschool Greek children, in relation to parental characteristics and region of residence. BMC Public Health 2007; 7:178.

4. White A., O'Brien B., Houlihan T. et al. Childhood obesity: parents fail to recognise, general practitioners fail to act. Ir Med J. 2012; 105(1):10-3.

5. Bouchard C. Childhood obesity: Are genetic differences involved? Am J ClinNutr 2009; 89: 1494S-1501S.

6. Dev D.A., McBride B.A., Fiese B.H. Risk Factors for Overweight/ Obesity in Preschool Children: An Ecological Approach. Child Obes 2013; 9 (5): 399-408.

7. Janssen I., Katzmarzyk P.T., Boyce W.F. et al. Comparison of overweight and obesity prevalence in schoolaged youth from 34 countries and their relationships with physical activity and dietary patterns. Obes Rev 2005; 6: 123-132.

8. Hill J.O., Wyatt H.R. Role of physical activity in preventing and treating obesity. J App/Physiol 2005; 99: 765-770.

9. Yu Z.B., Han S.P., Zhu G.Z. et al. Birth weight and subsequent risk of obesity: a systematic review and meta-analysis. Obes Rev. 2011; 12(7):525-542.

10. Oldroyd J., Renzaho A., Skouteris H. Low and high birth weight as risk factors for obesity among 4 to 5 -year-old Australian children: does gender matter? Eur J Pediatr. 2011; 170(7):899-906.

11. Y. Qiao, J. Ma, Y. Wang, W. Li et al. Birth weight and childhood obesity: a 12-country study. Int J Obes, 2015, Suppl 5: S74-S79.

12. Rossi C.E., de Assis Guedes de Vasconcelos F. Birth weight and obesity in children and adolescents: a systematic review. Rev Bras Epidemiol. 2010; 13(2): 1-13.

13. Freemark Michael. Pediatric Obesity Etiology, Pathogenesis, and Treatment, Springer 1st Edition, XV, 2010.

14. Nguyen V.T., Larson D.E., Johnson R.K., Goran M.L. Fat intake and adiposity in children of lean and obese parents. Am J Clin Nutr 1996; 63: $507-513$.

15. Maffeis C., Provera S., Filippi L. et al. Distribution of food intake as a risk factor for childhood obesity. Int J Obes 2000; 24: 75-80.

16. Zong X.N., Li H., Zhang Y.Q. Family-related risk factors of obesity among preschool children: results from a series of national epidemiological surveys in China. BMC Pub Health 2015; 15: 927-930.

17. Veena Mazarello Paes, Ken K. Ong, Rajalakshmi Lakshman. Factors influencing obesogenic dietary intake in young children ( $0-6$ years): 
systematic review of qualitative evidence. BMJ Open 2015; 5(9): e007396.

18. Moreno L.A., Rodriguez G. Dietary risk factors for development of childhood obesity. Curr Opin Clin Nutr Metab Care 2007; 10: 336-341.

19. Tucker L.A., Seljaas G.T., Hager R.L. Body fat percentage of children varies according to their diet composition. J Am Diet Assoc 1997; 97(9): 981- 986.

20. McCambridge T.M., Bernhardt D.T., Brenner J.S. et al. Active healthy living: Prevention of childhood obesity through increased physical activity. Pediatrics 2006; 117(5): 1834-1842.
21. Kreichauf S., Wildgruber A., Krombholz H. et al. Critical narrative review to identify educational strategies promoting physical activity in preschool. Obes Rev 2012; 13(1): 96-105.

22. Skouteris H., McCabe M., Swinburn B. et al. Parental influence and obesity prevention in pre-schoolers: a systematic review of interventions. Obes Rev 2011; 12(5): 315-328. 